\title{
Time-Optimal Trajectory Planning for Flexible Joint Robots
}

\author{
Alessandro Palleschi ${ }^{1}$, Riccardo Mengacci ${ }^{1}$, Franco Angelini ${ }^{1,2}$, \\ Danilo Caporale ${ }^{1}$, Lucia Pallottino ${ }^{1}$, Alessandro De Luca ${ }^{3}$, Manolo Garabini ${ }^{1}$
}

\begin{abstract}
In this paper, a new approach is proposed to optimally plan the motion along a parametrized path for flexible joint robots, i.e., robots whose structure is purposefully provided with compliant elements. State-of-the-art methods efficiently solve the problem in case of torque-controlled rigid robots via a translation of the optimal control problem into a convex optimization problem. Recently, we showed that, for jerk-controlled rigid robots, the problem could be recast into a non-convex optimization problem. The non-convexity is given by bilinear constraints that can be efficiently handled through McCormick relaxations and spatial Branch-and-Bound techniques. In this paper, we show that, even in case of robots with flexible joints, the time-optimal trajectory planning problem can be recast into a non-convex problem in which the non-convexity is still given by bilinear constraints. We performed experimental tests on a planar $2 \mathrm{R}$ elastic manipulator to validate the benefits of the proposed approach. The scalability of the method for robots with multiple degrees of freedom is also discussed.
\end{abstract}

Index Terms-Motion and Path Planning; Optimization and Optimal Control; Flexible Robots

\section{INTRODUCTION}

$\mathbf{R}$ ECENT years have seen an increasing interest in the use of robotics solutions in a new range of areas, e.g., healthcare, inspection and maintenance of infrastructure, agrifood, and agile production in the context of the Industry 4.0 initiative. These application areas require the robot to operate and interact efficiently with highly dynamic and unstructured environments, a complete paradigm shift for robots typically employed in conventional industrial applications, e.g., pick-and-place operations in structured, static and isolated environments. To allow energy efficient solutions and safe interactions with the environment, researchers have focused on the development of a new generation of lightweight robots

Manuscript received: September, 11, 2019; Revised December, 7, 2019; Accepted December, 30, 2019.

This paper was recommended for publication by Editor Nancy Amato upon evaluation of the Associate Editor and Reviewers' comments.

This work was supported in part by the European Unions Horizon 2020 research and innovation program as part of the projects ILIAD (Grant no. 732737) and THING (Grant no. 780883), and in part by the Italian Ministry of Education and Research in the framework of the CrossLab project (Departments of Excellence).

${ }^{1}$ Research Center "Enrico Piaggio", Dipartimento di Ingegneria dell'Informazione, University of Pisa, Largo Lucio Lazzarino 1, 56126 Pisa, Italy

alessandro.palleschi@phd.unipi.it

${ }^{2}$ Soft Robotics for Human Cooperation and Rehabilitation, Fondazione Istituto Italiano di Tecnologia, via Morego, 30, 16163 Genova, Italy

${ }^{3}$ Dipartimento di Ingegneria Informatica, Automatica e Gestionale, Sapienza Università di Roma, Via Ariosto 25, 00185 Roma, Italy

Digital Object Identifier (DOI): see top of this page.

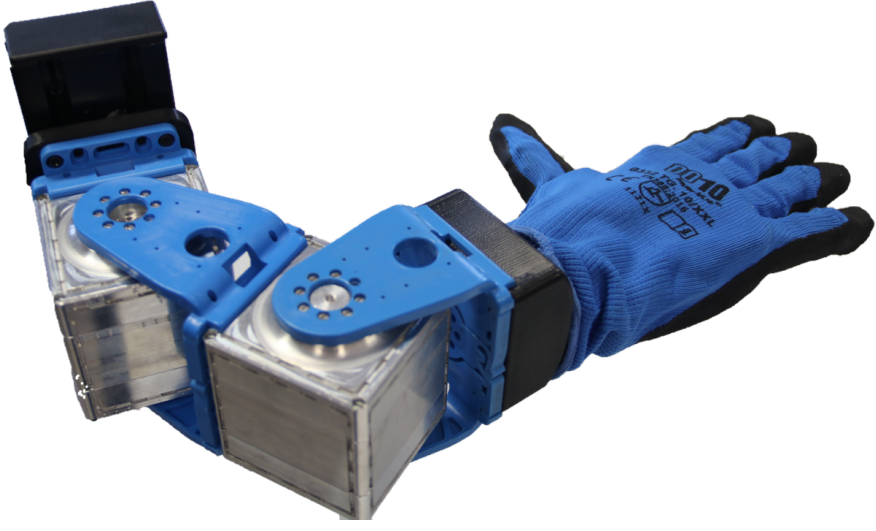

Fig. 1. Planar $2 \mathrm{R}$ manipulator, powered by two series elastic actuators and equipped with a soft end-effector.

capable of achieving the necessary level of compliance, i.e. soft robots. The interested reader can refer to [1] for a survey about soft robots with deformable bodies, or to [2] for robots with elastic elements lumped at the joint level.

It is well known that this higher degree of compliance comes at the cost of a more complex dynamic model and raises challenges for planning and control. In this work, we focus our attention on soft robots that use compliant elements in the actuation of the joints, i.e., flexible joint robots. Because of the advantages given by the use of compliant elements for the actuation, e.g., adaptability and resilience, soft robotic platforms are becoming more and more popular. Some examples are the quadrupedal robot ANYmal [3] and the humanoid WalkMan [4]. This spreading has made it necessary to develop algorithms capable of exploiting these elastic actuation units to their full potential, in order to guarantee a certain level of performance. In the literature, several works adopt optimal control methods to exploit the compliance of the actuators, where the reference trajectory is obtained formulating a constrained optimal control problem (OCP) [5], and closed-loop trajectory tracking is performed by nonlinear predictive controllers [6] or iterative linear quadratic regulators [7].

From the planning point of view, numerical optimization approaches are potentially able to simultaneously optimize the trajectory and the impedance of the robot. Recent works, e.g., [8], [9], have shown that the combination of offline and online optimization phases may reduce the computational requirements, allowing online optimal motion planning. These methods use a trajectory library approach, where a library of optimal parametric trajectories is generated offline. During 
motion, an online search of the best candidate solution for the current task parameters is performed, together with a refinement of the chosen solution.

However, to fully exploit the potential of flexible joint robots, several challenges have still to be solved. A prominent open problem tackled in this paper is the time-optimal trajectory planning for flexible joint robots. This problem has been widely investigated for torque-controlled rigid robots, with state-of-the-art methods that efficiently solve it through convex optimization techniques, see, e.g., [10]-[13].

These approaches reduce the complexity of the problem, by splitting it into two phases. In the first phase a reference path for the robot is generated, while a second tracking phase is used to optimize the motion along this path. Recently, we have shown that the time-optimal trajectory planning problem for rigid manipulator with joint constraints up to the jerk can be formulated as a non-convex optimization problem with bilinear and quadratic constraints [14].

Unfortunately, the existing optimal planning methods are not suitable for robots with compliant actuation, since they only consider the link dynamics, neglecting crucial actuation constraints such as the ones on the motors and elastic torques, which are likely to be violated. Indeed, these planning algorithms allow to constrain the link motion up to the acceleration/torque level. Rigid-based design applied to elastic robots may lead to inaccurate trajectories, a safety stop for the robot, or, in the worst case, to damage of the elastic actuators.

An extension of these planning algorithms for soft robots is challenging, since in general it would require the use of a more complex dynamics, due to the presence of elastic elements. The increase in complexity might be however balanced by the capability of guaranteeing a faster and feasible motion.

A possible approach is the one proposed in [15], for manipulators with only one flexible joint and based on a combination of flexible and rigid body models. The flexibility is considered only to smooth away acceleration discontinuities. Conversely, we present here a time-optimal trajectory planning algorithm for robots with multiple flexible joints and capable of considering and satisfying constraints on both the link and the motor variables.

The main contribution of the paper is the translation of the minimum-time optimization problem with the inclusion of the complete dynamics of the flexible joint robot into a non-convex Nonlinear Programming (NLP) problem. We show that, by defining a set of suitable nonlinear change of variables, it is possible to formulate a problem where the nonconvexity is given by bilinear and quadratics constraints only, which allow, in turn, for an efficient convex relaxation using McCormick envelopes [16].

To show the benefit and the effectiveness of our approach, experimental results using a $2 \mathrm{R}$ elastic manipulator, see Fig. 1, tracking a specified Cartesian trajectory are presented, together with considerations about the scalability of the method for robots with more degrees of freedom.

\section{PROBLEM FORMULATION}

The objective is to optimally plan the motion of a flexible joint robot that has to follow a sufficiently smooth parametrized path, specified for the link coordinates, $\boldsymbol{q}=$ $\boldsymbol{q}(s) \in \mathbb{R}^{n}$, where $n$ is the number of links of the robot. To formulate the optimization problem we exploit the possibility to parametrize the motion along a path using a scalar coordinate $s=s(t)$ and its time derivatives, as already presented in many works, e.g., [10] and [13]. For the rest of the paper we will use the Lagrange's notation, i.e., $x^{\prime}$, to denote the derivative of the variable $x$ w.r.t. $s$ and the Newton's notation, i.e., $\dot{x}$, to denote the time derivatives of the variable $x$. With this parametrization, we shall see how it is possible to write the optimal rest-to-rest trajectory planning problem for a flexible joint robot subject to constraints on both link and motor variables as

$$
\left\{\begin{array}{l}
\min _{s(\cdot)} \quad \int_{0}^{T} 1 \mathrm{~d} t \\
\text { subject to } \\
s(0)=0 \text { and } s(T)=1 \\
\dot{s}(0)=0 \text { and } \dot{s}(T)=0 \\
\ddot{s}(0)=0 \text { and } \ddot{s}(T)=0 \\
\dddot{s}(0)=0 \text { and } \dddot{s}(T)=0 \\
\dot{s}(t) \geq 0 \\
\dot{\boldsymbol{q}} \leq \dot{\boldsymbol{q}}(s(t)) \leq \overline{\dot{\boldsymbol{q}}} \\
\ddot{\ddot{\boldsymbol{q}}} \leq \ddot{\boldsymbol{q}}(s(t)) \leq \overline{\ddot{\boldsymbol{q}}} \\
\underline{\boldsymbol{\theta}} \leq \boldsymbol{\theta}(s(t)) \leq \overline{\boldsymbol{\theta}} \\
\dot{\boldsymbol{\theta}} \leq \dot{\boldsymbol{\theta}}(s(t)) \leq \overline{\boldsymbol{\theta}} \\
\underline{\boldsymbol{\tau}}_{\mathrm{e}} \leq \boldsymbol{\tau}_{\mathrm{e}}(s(t)) \leq \overline{\boldsymbol{\tau}}_{\mathrm{e}} \\
\underline{\boldsymbol{\tau}}_{\mathrm{m}} \leq \boldsymbol{\tau}_{\mathrm{m}}(s(t)) \leq \overline{\boldsymbol{\tau}}_{\mathrm{m}} \\
\text { for } t \in[0, T],
\end{array}\right.
$$

where $\dot{\boldsymbol{q}}$ and $\ddot{\boldsymbol{q}}$ are the vectors of velocities and accelerations of the links, $\boldsymbol{\theta}$ and $\dot{\boldsymbol{\theta}}$ are the vectors of angles and angular velocities of the motors, while $\tau_{\mathrm{m}}$ and $\tau_{\mathrm{e}}$ are the vectors of the motor and elastic torques, respectively. We use $\underline{x}$ and $\bar{x}$ to indicate the lower and upper bounds of the variable $x$, respectively. For simplicity, the presented formulation considers constant lower and upper bounds. However, the problem could still be formulated considering the dependency of the bounds on the path coordinate. For the sake of clarity, the time dependency of $s$ and its derivatives and the dependency on $s$ of the motor torques $\tau_{\mathrm{m}}$, the elastic torques $\tau_{\mathrm{e}}$, the link coordinates $\boldsymbol{q}$, the motor position $\boldsymbol{\theta}$ and their derivatives are omitted for the rest of the paper.

Our formulation is able to use information about the elasticity of the robot including the complete dynamics of the robot directly into the optimization.

Problem (1) can be reformulated in order to be parametrized by $s$ defining the change of variables

$$
\left\{\begin{array}{l}
b \triangleq \dot{s}^{2}, \alpha_{1} \triangleq b^{\prime}=2 \ddot{s}, \\
\alpha_{2} \triangleq b^{\prime \prime}=2 \frac{\dddot{s}}{\dot{s}}, \alpha_{3} \triangleq b^{\prime \prime \prime}=2 \frac{\left(s^{(4)} \dot{s}-\dddot{s} \dddot{s}\right)}{\dot{s}^{3}},
\end{array}\right.
$$

with $s^{(4)}=\mathrm{d}^{4} s / \mathrm{d} t^{4}$. It is worth noting that setting $\dot{s}(0)=$ $\dot{s}(T)=0$ does not result in a singularity, since also $\ddot{s}$ and $\dddot{s}$ are equal to zero at the motion boundary instants. In the open interval $] 0, T$, we can assume that $\dot{s}(t)>0$, which will be definitely enforced by the given nature of our 
problem, i.e., a minimum-time optimization problem. Under this hypothesis, the change of variables is always defined. The definition of these variables is convenient for the formulation of the optimization problem as discussed in more detail in the following, where we present a way to write the inequality constraints in (1). The reformulation of the objective function using the path coordinate $s$ is the same as the one proposed in [11] and [14], and it is not reported here.

\section{A. Inequality Constraints}

The proposed formulation permits to introduce constraints both on the link and the motor variables. Limits on the velocity and acceleration of the links can be included using the same formulation presented in [11] and [14], therefore are not reported here.

In order to write the motor-related constraints in terms of $s$, we used the following dynamic model of a robot with flexible joints presented by Spong [17]

$$
\left\{\begin{array}{l}
\boldsymbol{M}(\boldsymbol{q}) \ddot{\boldsymbol{q}}+\boldsymbol{C}(\boldsymbol{q}, \dot{\boldsymbol{q}}) \dot{\boldsymbol{q}}+\boldsymbol{G}(\boldsymbol{q})+\boldsymbol{K}(\boldsymbol{q}-\boldsymbol{\theta})=\mathbf{0} \\
\boldsymbol{B}_{\mathrm{m}} \ddot{\boldsymbol{\theta}}+\boldsymbol{K}(\boldsymbol{\theta}-\boldsymbol{q})=\tau_{\mathrm{m}},
\end{array}\right.
$$

where $\boldsymbol{M}, \boldsymbol{C}$ and $\boldsymbol{G}$ are the link inertia matrix, Coriolis and centrifugal matrix and gravity vector respectively, $\boldsymbol{K}$ is the constant diagonal joint stiffness matrix and $\boldsymbol{B}_{\mathrm{m}}$ is the constant diagonal motor inertia matrix.

On the motor side of the flexible transmission, we included constraints on position, velocity and motor torques, as well as on the elastic torques, i.e., $\tau_{\mathrm{e}}=\boldsymbol{K}(\boldsymbol{q}-\boldsymbol{\theta})$. From a practical point of view, in case of constant stiffness, the latter constraint limits the elastic deflection. This might be useful to reduce the risk of damaging the elastic elements of the actuation units.

1) Motor Torque Constraints: Equations (2)-(3) can be manipulated to retrieve the motor torques $\tau_{\mathrm{m}}$ as a function of the link variables only. The link dynamics (2) can be written in terms of $s$ similar to [11]

$$
\boldsymbol{m}(s) \ddot{s}+\boldsymbol{c}(s) \dot{s}^{2}+\boldsymbol{g}(s)+\boldsymbol{K}(\boldsymbol{q}(s)-\boldsymbol{\theta}(s))=\mathbf{0} .
$$

Differentiating twice (4) w.r.t. time it holds

$$
\begin{aligned}
& \frac{\mathrm{d}^{2}}{\mathrm{~d} t^{2}}(\boldsymbol{M}(\boldsymbol{q}) \ddot{\boldsymbol{q}}+\boldsymbol{C}(\boldsymbol{q}, \dot{\boldsymbol{q}}) \dot{\boldsymbol{q}}+\boldsymbol{G}(\boldsymbol{q})+\boldsymbol{K}(\boldsymbol{q}-\boldsymbol{\theta}))=\mathbf{0}, \\
& \Leftrightarrow \boldsymbol{m} s^{(4)}+2\left(\boldsymbol{m}^{\prime}+\boldsymbol{c}\right) \dddot{s} \dot{s}+\left(\boldsymbol{m}^{\prime \prime}+5 \mathbf{c}^{\prime}\right) \ddot{s} \dot{s}^{2}+ \\
& +\left(\boldsymbol{m}^{\prime}+2 \mathbf{c}\right) \ddot{s}^{2}+\boldsymbol{c}^{\prime \prime} \dot{s}^{4}+\boldsymbol{g}^{\prime \prime} \dot{s}^{2}+\boldsymbol{g}^{\prime} \ddot{s}+\boldsymbol{K}(\ddot{\boldsymbol{q}}-\ddot{\boldsymbol{\theta}})=\mathbf{0} .
\end{aligned}
$$

Knowing that $\ddot{\boldsymbol{\theta}}=\boldsymbol{B}_{\mathrm{m}}^{-1}\left(\boldsymbol{\tau}_{\mathrm{m}}-\boldsymbol{K}(\boldsymbol{\theta}-\boldsymbol{q})\right), \boldsymbol{K}(\boldsymbol{\theta}-\boldsymbol{q})=\boldsymbol{M} \ddot{\boldsymbol{q}}+$ $\boldsymbol{C} \dot{\boldsymbol{q}}+\boldsymbol{G}$ and $\ddot{\boldsymbol{q}}=\boldsymbol{q}^{\prime} \ddot{s}+\boldsymbol{q}^{\prime \prime} \dot{s}^{2}$ we can rewrite (5) as

$$
\begin{aligned}
\boldsymbol{\Gamma} \boldsymbol{\tau}_{\boldsymbol{m}}= & \boldsymbol{k}_{0} b^{\prime \prime \prime} b+\boldsymbol{k}_{1} b^{\prime \prime} b^{\prime}+\boldsymbol{k}_{2} b^{\prime \prime} b+\boldsymbol{k}_{3} b^{\prime} b+ \\
& +\boldsymbol{k}_{4}\left(b^{\prime}\right)^{2}+\boldsymbol{k}_{5} b^{\prime}+\boldsymbol{k}_{6} b^{2}+\boldsymbol{k}_{7} b+\boldsymbol{k}_{8}= \\
= & \boldsymbol{k}_{0} \beta_{1}+\boldsymbol{k}_{1} \beta_{2}+\boldsymbol{k}_{2} \beta_{3}+\boldsymbol{k}_{3} \beta_{4}+ \\
& +\boldsymbol{k}_{4} \beta_{5}+\boldsymbol{k}_{5} \alpha_{1}+\boldsymbol{k}_{6} \beta_{6}+\boldsymbol{k}_{7} b+\boldsymbol{k}_{8},
\end{aligned}
$$

where we have defined $\boldsymbol{\Gamma} \triangleq \boldsymbol{K} \boldsymbol{B}_{\mathrm{m}}^{-1}$ and

$$
\begin{gathered}
\beta_{1} \triangleq b^{\prime \prime \prime} b, \beta_{2} \triangleq b^{\prime \prime} b^{\prime}, \beta_{3} \triangleq b^{\prime \prime} b, \beta_{4} \triangleq b^{\prime} b, \\
\beta_{5} \triangleq\left(b^{\prime}\right)^{2}, \beta_{6} \triangleq b^{2},
\end{gathered}
$$

and where the vectors $\boldsymbol{k}_{i}$ are only functions of the path coordinate $s$

$$
\begin{gathered}
\boldsymbol{k}_{0} \triangleq \boldsymbol{m} / 2, \boldsymbol{k}_{1} \triangleq \boldsymbol{m} / 4, \boldsymbol{k}_{2} \triangleq\left(\boldsymbol{m}^{\prime}+\boldsymbol{c}\right), \\
\boldsymbol{k}_{3} \triangleq\left(\boldsymbol{m}^{\prime \prime}+5 \boldsymbol{c}\right) / 2, \boldsymbol{k}_{4} \triangleq\left(\boldsymbol{m}^{\prime}+2 \boldsymbol{c}\right) / 4, \\
\boldsymbol{k}_{5} \triangleq \boldsymbol{g}^{\prime \prime}+\boldsymbol{K}\left(\boldsymbol{q}^{\prime}+\boldsymbol{B}_{\mathrm{m}}^{-1} \boldsymbol{m}\right) / 2, \boldsymbol{k}_{6} \triangleq \boldsymbol{c}^{\prime \prime}, \\
\boldsymbol{k}_{7} \triangleq \boldsymbol{g}^{\prime \prime}+\boldsymbol{K}\left(\boldsymbol{q}^{\prime \prime}+\boldsymbol{B}_{\mathrm{m}}^{-1} \boldsymbol{c}\right), \boldsymbol{k}_{8} \triangleq \boldsymbol{K} \boldsymbol{B}_{\mathrm{m}}^{-1} \boldsymbol{g} .
\end{gathered}
$$

With this change of variables, the motor torques are now affine in the variables $\beta_{i}$ and $\alpha_{i}$, and the torque constraints can be written as

$$
\begin{aligned}
\boldsymbol{\Gamma} \underline{\boldsymbol{\tau}}_{\mathrm{m}} \leq & \boldsymbol{k}_{0} \beta_{1}+\boldsymbol{k}_{1} \beta_{2}+\boldsymbol{k}_{2} \beta_{3}+\boldsymbol{k}_{3} \beta_{4}+ \\
& +\boldsymbol{k}_{4} \beta_{5}+\boldsymbol{k}_{5} \alpha_{1}+\boldsymbol{k}_{6} \beta_{6}+\boldsymbol{k}_{7} b+\boldsymbol{k}_{8} \leq \boldsymbol{\Gamma} \overline{\boldsymbol{\tau}}_{\mathrm{m}} .
\end{aligned}
$$

2) Motor Position Constraints: Since we want to follow a specified path for the link coordinates, it is necessary to constrain the resulting trajectory followed by the motors, to avoid exceeding their position limits.

Given model (2)-(3) of a series elastic robot, it is possible to compute the motor angles from (2)

$$
\boldsymbol{\theta}=\boldsymbol{q}+\boldsymbol{K}^{-1}(\boldsymbol{M}(\boldsymbol{q}) \ddot{\boldsymbol{q}}+\boldsymbol{C}(\boldsymbol{q}, \dot{\boldsymbol{q}}) \dot{\boldsymbol{q}}+\boldsymbol{G}(\boldsymbol{q})) .
$$

Using (7), position constraints can be included using

$$
\begin{aligned}
& \underline{\boldsymbol{\theta}} \leq \boldsymbol{\theta} \leq \overline{\boldsymbol{\theta}}, \\
\Leftrightarrow & \underline{\boldsymbol{\theta}} \leq \boldsymbol{q}+\boldsymbol{k}_{9} b^{\prime}+\boldsymbol{k}_{10} b+\boldsymbol{K}^{-1} \boldsymbol{g} \leq \overline{\boldsymbol{\theta}},
\end{aligned}
$$

where $\boldsymbol{k}_{9}$ and $\boldsymbol{k}_{10}$ are only functions of $s$, i.e., $\boldsymbol{k}_{9} \triangleq$ $\boldsymbol{K}^{-1} \boldsymbol{m} / 2, \boldsymbol{k}_{10} \triangleq \boldsymbol{K}^{-1} \boldsymbol{c}$.

3) Velocity Constraints: The velocity of the motors can be obtained differentiating (7) w.r.t. time. Then, if there exists an upper bound $\overline{\dot{\theta}}$ and a lower bound $\underline{\dot{\theta}}$ on the velocities, it is possible to write the following inequalities

$$
\begin{aligned}
\dot{\dot{\boldsymbol{\theta}}} \leq \dot{\boldsymbol{\theta}} \leq \overline{\dot{\boldsymbol{\theta}}} \\
\Leftrightarrow \underline{\dot{\boldsymbol{\theta}} \leq} \leq \dot{\boldsymbol{q}}+\boldsymbol{K}^{-1}\left(\boldsymbol{m} \dddot{s}+\left(\boldsymbol{m}^{\prime}+2 \boldsymbol{c}\right) \dddot{s} \dot{s}+\boldsymbol{c}^{\prime} \dot{s}^{3}+\boldsymbol{g}^{\prime} \dot{s}\right) \leq \overline{\boldsymbol{\theta}}, \\
\Leftrightarrow \dot{\boldsymbol{\theta}} \leq \sqrt{b}\left(\boldsymbol{q}^{\prime}+\boldsymbol{K}^{-1}\left(\boldsymbol{m} b^{\prime \prime} / 2+\left(\boldsymbol{m}^{\prime}+2 \boldsymbol{c}\right) b^{\prime} / 2+\right.\right. \\
\left.\left.\quad+\boldsymbol{c}^{\prime} b+\boldsymbol{g}^{\prime}\right)\right) \leq \overline{\dot{\boldsymbol{\theta}}} .
\end{aligned}
$$

We can introduce the following nonlinear change of variables $\beta_{7} \triangleq \sqrt{b}, \beta_{8} \triangleq b^{\prime \prime} \sqrt{b}, \beta_{9} \triangleq b^{\prime} \sqrt{b}, \beta_{10} \triangleq b \sqrt{b}$, and the inequality can be written as

$$
\dot{\boldsymbol{\theta}} \leq \boldsymbol{k}_{11} \beta_{7}+\boldsymbol{k}_{12} \beta_{8}+\boldsymbol{k}_{13} \beta_{9}+\boldsymbol{k}_{14} \beta_{10} \leq \overline{\dot{\boldsymbol{\theta}}}
$$

with the following definition of the vectors $\boldsymbol{k}_{i}$

$$
\begin{gathered}
\boldsymbol{k}_{11} \triangleq \boldsymbol{q}^{\prime}+\boldsymbol{K}^{-1} \boldsymbol{g}^{\prime}, \boldsymbol{k}_{12} \triangleq \boldsymbol{K}^{-1} \boldsymbol{m} / 2=\boldsymbol{k}_{9} \\
\boldsymbol{k}_{13} \triangleq \boldsymbol{K}^{-1}\left(\boldsymbol{m}^{\prime}+2 \boldsymbol{c}\right) / 2=2 \boldsymbol{K}^{-1} \boldsymbol{k}_{4}, \boldsymbol{k}_{14} \triangleq \boldsymbol{K}^{-1} \boldsymbol{c}^{\prime} .
\end{gathered}
$$

4) Elastic Torque Constraints: Other than the motor torque, it is possible to impose limits on the elastic torque of the system. Indeed, in presence of bounds $\bar{\tau}_{\mathrm{e}}$ and $\underline{\tau}_{\mathrm{e}}$ it is possible to write the following inequality

$$
\begin{aligned}
& \underline{\boldsymbol{\tau}}_{\mathrm{e}} \leq \boldsymbol{\tau}_{\mathrm{e}} \leq \overline{\boldsymbol{\tau}}_{\mathrm{e}}, \\
& \Leftrightarrow \underline{\boldsymbol{\tau}}_{\mathrm{e}} \leq \boldsymbol{K}(\boldsymbol{\theta}-\boldsymbol{q}) \leq \overline{\boldsymbol{\tau}}_{\mathrm{e}}, \\
& \Leftrightarrow \underline{\boldsymbol{\tau}}_{\mathrm{e}} \leq \boldsymbol{k}_{15} \alpha_{1}+\boldsymbol{k}_{16} b+\boldsymbol{g} \leq \overline{\boldsymbol{\tau}}_{\mathrm{e}}
\end{aligned}
$$

where the vectors $\boldsymbol{k}_{i}$ are again only functions of $s$, i.e., $\boldsymbol{k}_{15} \triangleq$ $\boldsymbol{m} / 2=\boldsymbol{k}_{1}, \boldsymbol{k}_{16} \triangleq \boldsymbol{c}$. 


\section{B. Optimization Problem Formulation}

Using (6), (8), (9) and (10) for the constraints, problem (1) can be reformulated as

$$
\begin{aligned}
& \min _{b(\cdot), \beta_{i}(\cdot), \alpha_{i}(\cdot)} \int_{0}^{1} \frac{1}{\sqrt{b}} \mathrm{~d} s \\
& \text { subject to } \\
& \boldsymbol{\Gamma} \boldsymbol{\tau}_{\mathrm{m}}=\boldsymbol{k}_{0} \beta_{1}+\boldsymbol{k}_{1} \beta_{2}+\beta_{3}+\boldsymbol{k}_{3} \beta_{4}+ \\
& +\boldsymbol{k}_{4} \beta_{5}+\boldsymbol{k}_{5} \alpha_{1}+\boldsymbol{k}_{6} \beta_{6}+\boldsymbol{k}_{7} b+\boldsymbol{k}_{8} \\
& b(0)=0 \text { and } b(1)=0 \\
& \alpha_{1}(0)=0 \text { and } \alpha_{1}(1)=0 \\
& \alpha_{2}(0) \sqrt{b(0)}=0 \text { and } \alpha_{2}(1) \sqrt{b(1)}=0 \\
& \alpha_{1}=b^{\prime}, \alpha_{2}=b^{\prime \prime}, \alpha_{3}=b^{\prime \prime \prime} \\
& \beta_{1}=\alpha_{3} b, \beta_{2}=\alpha_{2} \alpha_{1}, \beta_{3}=\alpha_{2} b, \beta_{4}=\alpha_{1} b \\
& \beta_{8}=\alpha_{2} \beta_{7}, \beta_{9}=\alpha_{1} \beta_{7}, \beta_{10}=b \beta_{7} \\
& \beta_{5}=\alpha_{1}^{2}, \beta_{6}=b^{2}, \beta_{7}^{2}=b \\
& 0 \leq b \leq \min _{i \in 1, n}\left(\overline{\dot{q}}_{i}\right)^{2} /\left(q_{i}^{\prime}\right)^{2} \\
& \ddot{\ddot{q}} \leq \boldsymbol{q}^{\prime \prime} b+\boldsymbol{q}^{\prime} \alpha_{1} / 2 \leq \overline{\ddot{q}} \\
& \underline{\boldsymbol{\theta}}-\boldsymbol{q}-\boldsymbol{K}^{-1} \boldsymbol{g} \leq \boldsymbol{k}_{9} \alpha_{1}+\boldsymbol{k}_{10} b \leq \overline{\boldsymbol{\theta}}-\boldsymbol{q}-\boldsymbol{K}^{-1} \boldsymbol{g} \\
& \underline{\dot{\boldsymbol{\theta}}} \leq \boldsymbol{k}_{11} \beta_{7}+\boldsymbol{k}_{12} \beta_{8}+\boldsymbol{k}_{13} \beta_{9}+\boldsymbol{k}_{14} \beta_{10} \leq \overline{\boldsymbol{\theta}} \\
& \underline{\boldsymbol{\tau}}_{\mathrm{e}} \leq \boldsymbol{k}_{15} \alpha_{1}+\boldsymbol{k}_{16} b \leq \overline{\boldsymbol{\tau}}_{\mathrm{e}} \\
& \underline{\tau}_{\mathrm{m}} \leq \boldsymbol{\tau}_{\mathrm{m}} \leq \overline{\boldsymbol{\tau}}_{\mathrm{m}} \\
& \text { for } s \in[0,1] \text {. }
\end{aligned}
$$

The problem is non-convex and with a set of nonlinear constraints (11)-(13) introduced by the set of variables $\beta_{i}$.

\section{Numerical Solution}

To find a numerical solution for the problem we can use a direct transcription approach. The path coordinate $s$ is discretized on $[0,1]$ with $N+1$ grid points $s_{0}=0 \leq s_{j}<=1=$ $s_{N}$ for $j=0, \cdots, N$. The functions $b(s)$ and the previously defined auxiliary functions $\alpha_{i}(s)$ and $\beta_{i}(s)$ are modeled by a finite number of variables, i.e., $b_{j}=b\left(s_{j}\right), \beta_{i j}=\beta_{i}\left(s_{j}\right)$ and $\alpha_{i j}=\alpha_{i}\left(s_{j}\right)$. The function $b(s)$ is modeled as piecewise cubic, since it has to be three times differentiable. For each interval $\left[s_{j}, s_{j+1}\right]$, we use a cubic Lagrange polynomial that interpolates $b_{j}, b_{j+1}, b_{j+2}$ and $b_{j+3}$. The structure of the discretized problem is similar to the one presented in [14], and it is not reported in its entirety. Some considerations can be made on the discretization of constraints (13), i.e., $\beta_{5 j}=\alpha_{1 j}^{2}, \beta_{6 j}=b_{j}^{2}$ and $\beta_{7 j}^{2}=b_{j}$, that are non-convex quadratic constraints, and of constraints (11)-(12)

$$
\begin{aligned}
& \beta_{1 j}=\alpha_{3 j} b_{j}, \beta_{2 j}=\alpha_{2 j} \alpha_{1 j}, \beta_{3 j}=\alpha_{2 j} b_{j}, \beta_{4 j}=\alpha_{1 j} b_{j}, \\
& \beta_{8 j}=\alpha_{2 j} \beta_{7 j}, \beta_{9 j}=\alpha_{1 j} \beta_{7 j}, \beta_{10 j}=b_{j} \beta_{7 j} .
\end{aligned}
$$

It is known that finding the global optimal solution for this type of large space optimization problems is, in general, not trivial. Often spatial Branch-and-Bound (sBB) methods are employed [18]. These techniques need to solve a relaxed form of the problem, where the non-convex elements are substituted by convex approximations [19], to find a lower bound of the optimal solution. The fact that the non-convex

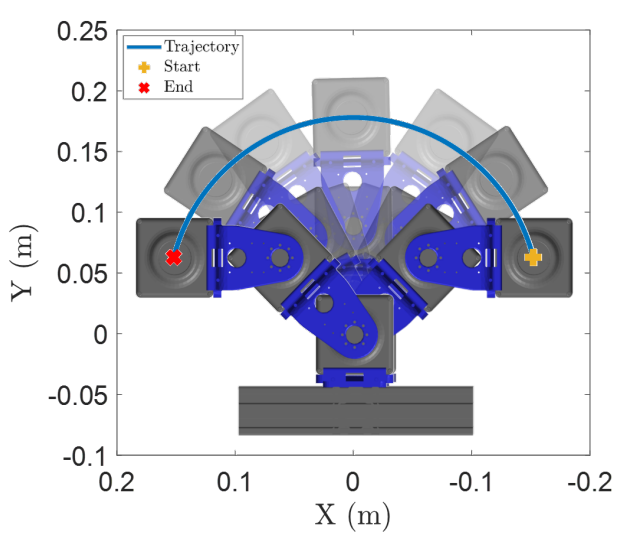

(a) Desired Cartesian trajectory to be tracked.

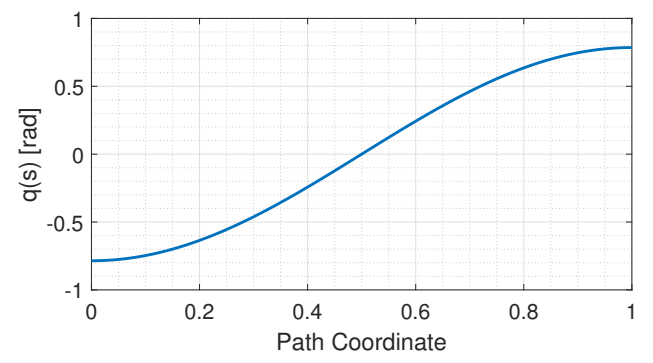

(b) Desired joint trajectory to be tracked.

Fig. 2. Cartesian and joint path to track. The two joints follow the same path, from $-\pi / 4$ to $\pi / 4$.

optimization problem can be rewritten using quadratic and bilinear constraints (11)-(13), as for the problem we presented in [14], makes it possible to use a convex relaxation provided by McCormick Envelopes [20]. Indeed, it allows to efficiently find the solution of the problem by tightening the gap between the lower bound solution and the optimal one.

\section{EXPERIMENTAL VALIDATION}

In this section, the model of the robot used to plan the trajectory and the bounds used in the optimization are described, together with a comparison of the results with the ones obtained using different planning techniques.

\section{A. Experimental Setup}

The soft robot used for the tests, showed in Fig. 1, is a planar 2R robot, powered by series elastic actuators (SEAs) like the ones used in [8]. The configuration of the system is defined with the joint angles $\boldsymbol{q}=\left[q_{1}, q_{2}\right]^{T}$ and with the motor angles $\boldsymbol{\theta}=\left[\theta_{1}, \theta_{2}\right]^{T}$. The stiffness of the two SEAs are $K_{1}=3.7092$ $\mathrm{Nm} / \mathrm{rad}$ and $K_{2}=2.1392 \mathrm{Nm} / \mathrm{rad}$. Each motor has an inertia equal to $B_{\mathrm{m} i}=0.0231 \mathrm{Nms}^{2}$.

The robot has to follow an assigned path in the Cartesian space, see Figure 2(a), that has been mapped in a path for the two joints showed in Figure 2(b). Both the joints follow the same path, the robot starts with $\boldsymbol{q}(0)=[-\pi / 4,-\pi / 4]^{T}$ and ends with $\boldsymbol{q}(T)=[\pi / 4, \pi / 4]^{T}$.

The bounds for each link have been set as $\overline{\dot{q}}_{i}=6 \mathrm{rad} / \mathrm{s}$, $\overline{\tilde{q}}_{i}=50 \mathrm{rad} / \mathrm{s}^{2}$, while for the motor we have defined realistic 

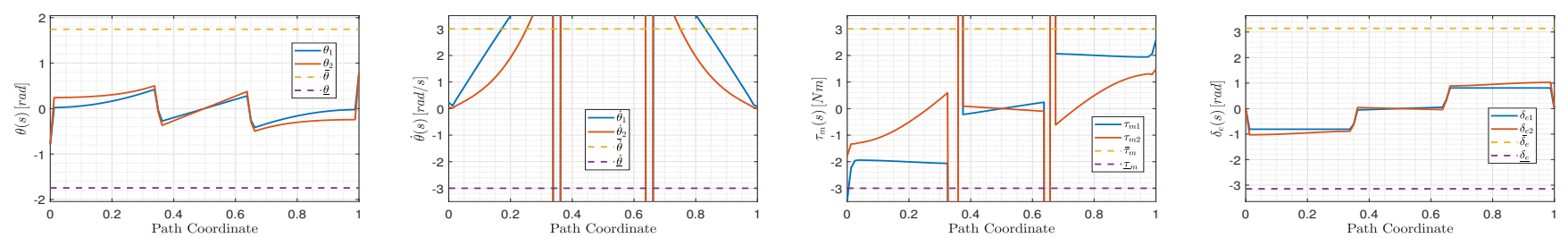

(a) Planned signals using rigid-based technique $\mathbf{R}$.
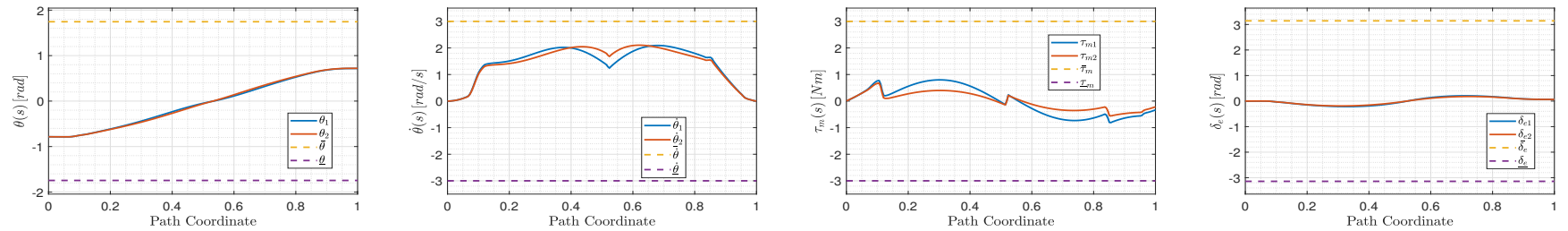

(b) Planned signals using snap-limited technique IC.
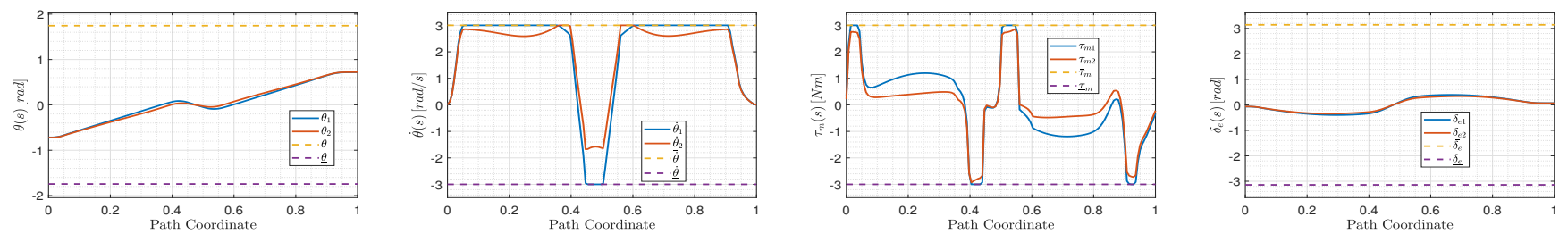

(c) Planned signals using our algorithm ED.

Fig. 3. From left to right: evolution of the planned motor angles, velocities, torques and of the elastic deflections for the three planning techniques $\mathbf{R}$, IC and ED. Solid line is the signal, while the dashed lines represent the lower and upper bounds.

bounds for the angles, velocities, torque and the elastic deflection $\delta_{\mathrm{e}_{i}} \triangleq q_{i}-\theta_{i}$, i.e., $\bar{\theta}_{i}=1.75 \mathrm{rad}, \dot{\theta}_{i}=3 \mathrm{rad} / \mathrm{s}, \bar{\tau}_{\mathrm{m}_{i}}=3 \mathrm{Nm}$ and $\bar{\delta}_{\mathrm{e}_{i}}=\pi$ rad. For simplicity, we we have used everywhere symmetric bounds, i.e., $\underline{x}=-\bar{x}$, for a constrained variable $x$.

The objective of the test is to evaluate the behavior of the elastic robot, in terms of the evolution of the motor angles, velocities and torques, when the parametrized link trajectory is used to generate the corresponding trajectories for the motor angles using (7). These angles are then used as a feedforward reference trajectory for the motors, which are position controlled.

In the following, the output of the planning algorithm and the experimental results tracking the planned trajectory with the real robot are presented, and we indicate our proposed approach using the name Elastic Dynamics (ED). Our results have been compared with those obtained using two different planning techniques. The first technique, indicated in the following with the name Rigid (R), is the one presented in [11]. This method employs only the rigid model of the robot to plan the trajectory. The second technique, named Integrators Chain (IC), employs a different formulation where the complete model of the elastic robot is not used in the optimization problem but instead considers an extension of the jerk-limited planning presented in our previous work [14] so as to include snap constraints. Details about this formulation are reported in the Appendix. The bounds for the jerk and the snap have been set as $\bar{q}_{i}=400 \mathrm{rad} / \mathrm{s}^{3}$ and $\bar{q}_{i}^{(4)}=500 \mathrm{rad} / \mathrm{s}^{4}$.
TABLE I

OPTIMAL TIME AND NORMALIZED PEAK VALUES FOR THE PLANNED MOTOR VARIABLES AND USING THE THREE TECHNIQUES

\begin{tabular}{|c|c|c|c|c|}
\hline & $\mathrm{T}$ & $\max _{i}\left|\theta_{i} / \bar{\theta}_{i}\right|$ & $\max _{i}\left|\dot{\theta}_{i} / \dot{\dot{\theta}}_{i}\right|$ & $\max _{i}\left|\tau_{m i} / \bar{\tau}_{m i}\right|$ \\
\hline $\mathbf{R}$ & 0.39 & 0.43 & 83 & 532 \\
\hline $\mathbf{I C}$ & 1.03 & 0.45 & 0.70 & 0.27 \\
\hline ED & 0.70 & 0.41 & 1.0 & 1.0 \\
\hline
\end{tabular}

\section{B. Planning Algorithm Results}

In this section, the results of the three planning strategies are reported. The optimization problem has been solved in MATLAB, using SCIP solver provided by YALMIP [21]. Table I shows the computed optimal time $T$, expressed in seconds, and the peak values for the motor variables, normalized w.r.t. their maximum values, for the three planning strategies, while in Fig. 3, the results of the trajectory planning using the three algorithms are shown. All the signals have been reported with the path parameter $s$ on the $\mathrm{x}$-axis and the bounds for each variable are displayed (dashed lines). Observing the data, we see how using classic rigid-body techniques the resulting motion of the robot is considerably faster but fails to meet the limitation on the motor velocities and torques. The snaplimited approach IC produces a slower motion, although it is able to satisfy the bounds on the motor variables. On the other hand, the inclusion of the elastic model at the planning stage using our approach produces a feasible but faster trajectory 

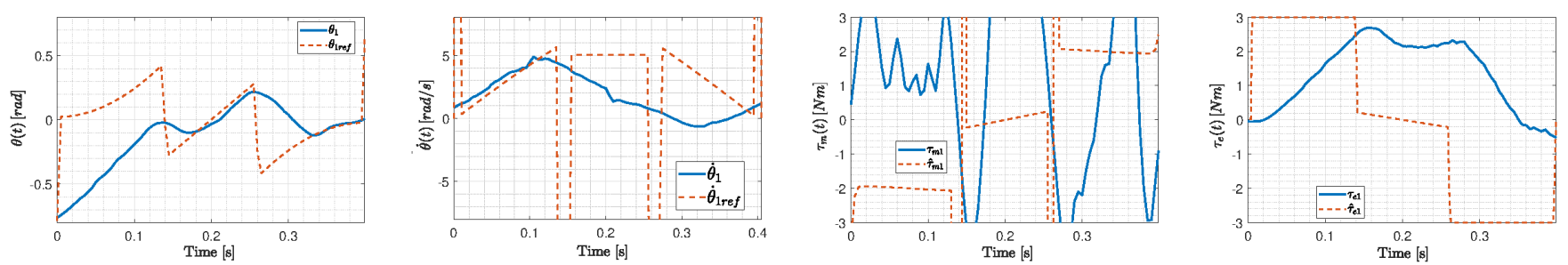

(a) First Joint: From left to right motor angle, velocity, torque and elastic torque. Solid line is the real signal, while dashed is the planned one.
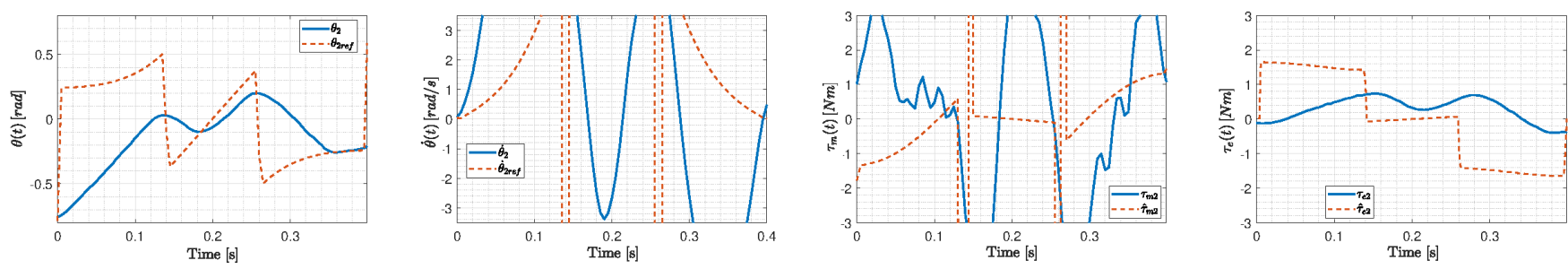

(b) Second Joint: From left to right motor angle, velocity, torque and elastic torque. Solid line is the real signal, while dashed is the planned one.

Fig. 4. Real robot tracking the trajectory planned with technique R.

than IC, although slower than the unfeasible one planned using R. Moreover, as expected for this type of optimization problems, using ED it can be seen in Fig. 6 that at least one of the motor velocities or torques takes its maximum or minimum value, at almost any time during motion (except for a few initial and final time instants). With respect to ED, the main limitation of the strategy IC is that one cannot impose constraints directly on motor velocities and torques. By constraining instead the link variables up to the snap, we may limit motor velocities and accelerations below their actual capabilities. As a matter of fact, the relationship between the link jerk/snap and the motor velocities/torques is not trivial in the general case. An incorrect setting of high-order but purely kinematic bounds on link motion may result in a poorer performance, for too conservative choices, or to unfeasible motion for the flexible joint robot, conversely. It is worth noting in Fig. 5 that motor velocities and torques never saturate at their bounds when using the IC method; accordingly, the chosen bounds for jerk and snap were indeed too strict.

\section{Real Robot}

In Figures 4, 5, and 6 are shown the evolution of the motor trajectories, velocities and torques and the elastic torques of the real robot executing the planned trajectories. For IC and ED, the differences between the planned evolution and the real one can be explained by differences between the model used to generate the trajectories and the real system. A possible source of modeling error is the presence of friction, which has been neglected in our robot model. In Table II are reported the trajectory time $T$ and the peak values for the motors variables normalized w.r.t. their upper bounds.

It is worth noting how using $\mathbf{R}$ the motors fail to track the given references and the resulting peak values for the motor velocities and torques are above the given bounds. As expected, not including the elasticity of the robot during planning does not allow to produce feasible trajectories for the
TABLE II

TRAJECTORY TIME AND NORMALIZED PEAK VALUES FOR THE MOTOR VARIABLES OF THE REAL ROBOT USING THE THREE TECHNIQUES

\begin{tabular}{|c|c|c|c|c|}
\hline & $\mathrm{T}$ & $\max _{i}\left|\theta_{i} / \bar{\theta}_{i}\right|$ & $\max _{i}\left|\dot{\theta}_{i} / \overline{\dot{\theta}}_{i}\right|$ & $\max _{i}\left|\tau_{m i} / \bar{\tau}_{m i}\right|$ \\
\hline $\mathbf{R}$ & 0.39 & 0.45 & 2.25 & 2.42 \\
\hline IC & 1.03 & 0.45 & 0.76 & 0.38 \\
\hline ED & 0.70 & 0.45 & 0.99 & 0.98 \\
\hline
\end{tabular}

motors, and, as in this case, the resulting motion is different from the planned one. Using the trajectories planned with IC and ED the values are instead within the bounds, but with ED we can obtain a faster motion since we are able to better exploit the actuation capabilities.

\section{Computational Time and Scalability for More Degrees of Freedoms}

The proposed approach has been tested on a $2 \mathrm{R}$ robot, but the formulation can also be extended for more complex systems. However, the non-convexity of the problem makes computationally demanding to numerically find the global optimum. In this section a brief discussion on the computational time of the algorithm and the scalability of the method for more complex robots are reported At the current state, for the given $2 \mathrm{R}$ robot and its bounds, discretizing the path using 150 grid points, using the MATLAB implementation of SCIP as solver on a Laptop PC equipped with Intel Core i7 Processor $(6 \times 2.20 \mathrm{GHz})$ and $16 \mathrm{~GB}$ DDR4 RAM, it is possible to find the optimal solution in about $210 \mathrm{~s}$ This result clearly shows how, at this stage, the algorithm can only be used as an offline planner To demonstrate the feasibility of our approach to more general robotic systems, we used our algorithm to plan the trajectory of a 7-DoF flexible joint manipulator, where each link has to follow the same trajectory presented in Section III Using the same bounds for the variables and the same 

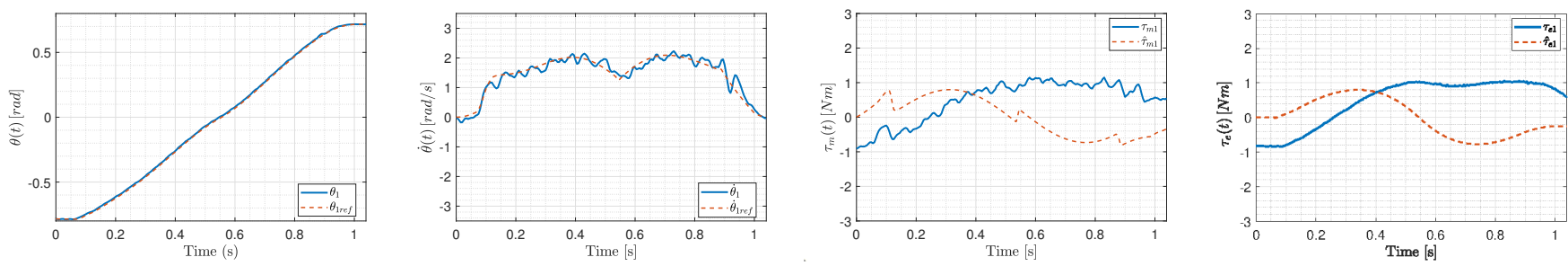

(a) First Joint: From left to right motor angle, velocity, torque and elastic torque. Solid line is the real signal, while dashed is the planned one.
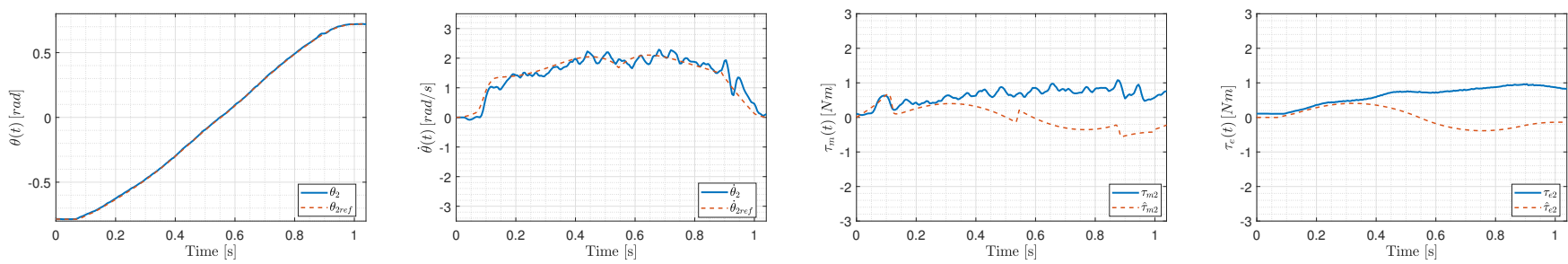

(b) Second Joint: From left to right motor angle, velocity, torque and elastic torque. Solid line is the real signal, while dashed is the planned one.

Fig. 5. Real robot tracking the trajectory planned with technique IC.
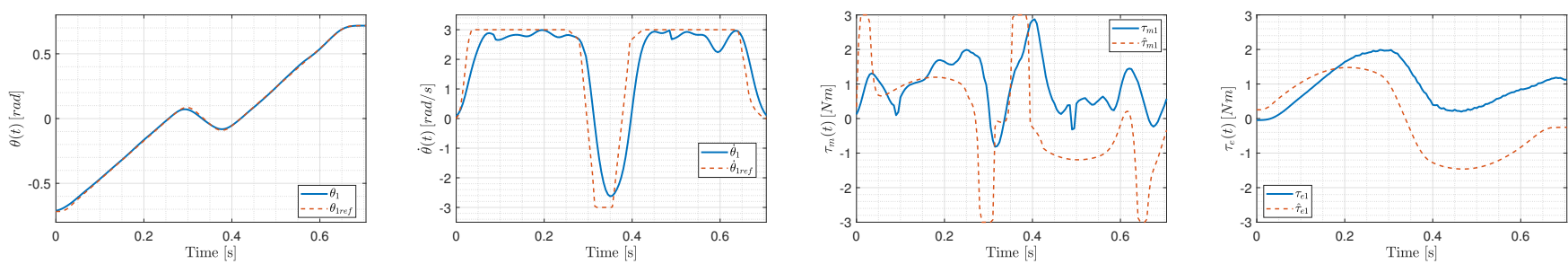

(a) First Joint: From left to right motor angle, velocity, torque and elastic torque. Solid line is the real signal, while dashed is the planned one.
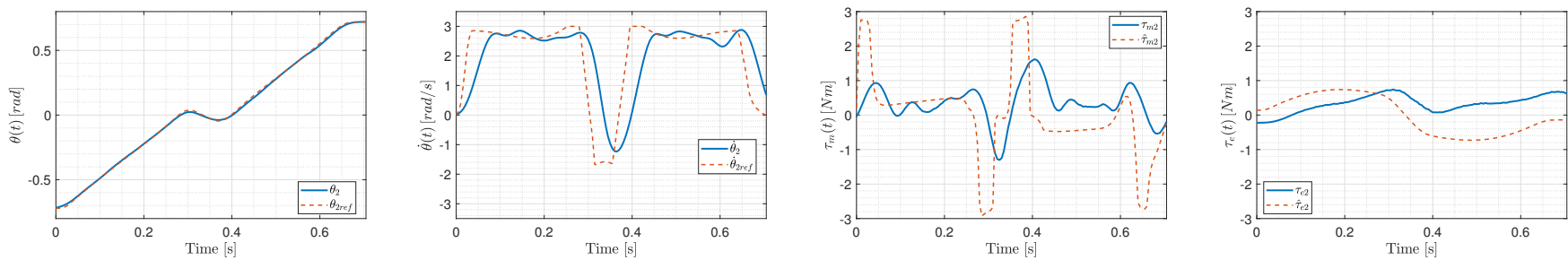

(b) Second Joint: From left to right motor angle, velocity, torque and elastic torque. Solid line is the real signal, while dashed is the planned one.

Fig. 6. Real robot tracking the trajectory planned with technique ED.

number of steps to discretize the problem, the algorithm was able to found a solution in about $270 \mathrm{~s}$, quite close to the time needed for the $2 \mathrm{R}$ robot despite the increased number of degrees of freedom. This result could be justified by the particular formulation of the problem and by the form we used to define constraints, but a more in-depth analysis about this feature is deferred to future works.

\section{CONCLUSIONS}

In this paper, we presented a time-optimal trajectory planning algorithm for moving a flexible joint robot along smooth parametrized paths. We have shown that the problem can be translated into a non-convex NLP with bilinear and quadratic constraints, and then solved by efficient numerical techniques. The experimental tests carried out on a $2 \mathrm{R}$ manipulator with SEA actuation demonstrate the effectiveness of the approach, capable of generating feasible and time-optimal reference trajectories for the robot actuators. Furthermore, we reported considerations about the scalability of the proposed approach, presenting results for the trajectory planning on a 7-DoF robot. Future works will try to extend this approach to robots powered by variable stiffness actuators (VSAs) and to flexible link soft robots, a problem that has been already studied, see, e.g., [22], but still with many open problems.

\section{APPENDIX}

In Section III, we compared our approach with a planning algorithm that considers only constraints on the link coordinates derivatives. By constraining the snap and the jerk of the links we can limit the velocities and accelerations of the motors. The importance of including constraints on higher derivatives of the link coordinates has been already studied 
in the literature, see e.g. [23], but the problem is in general complex. In our previous work [14] we presented a timeoptimal trajectory planning algorithm considering constraints up to the jerk. To include snap constraints we can express it in terms of $s$

$$
\boldsymbol{q}^{(4)}=\boldsymbol{q}^{\prime} s^{(4)}+\boldsymbol{q}^{\prime \prime}\left(3 \ddot{s}^{2}+4 \dddot{s} \dddot{s}\right)+6 \boldsymbol{q}^{\prime \prime \prime} \ddot{s} \dot{s}^{2}+\boldsymbol{q}^{\mathrm{iv}} \dot{s}^{4} .
$$

Using the change of variables (1) it is possible to write the jerk and snap equations as

$$
\left\{\begin{aligned}
\dddot{\boldsymbol{q}}= & \boldsymbol{q}^{\prime} \alpha_{2} \sqrt{b} / 2+(3 / 2) \boldsymbol{q}^{\prime \prime} \alpha_{1} \sqrt{b}+\boldsymbol{q}^{\prime \prime \prime} b \sqrt{b} \\
\boldsymbol{q}^{(4)}= & \alpha_{3} b \boldsymbol{q}^{\prime} / 2+2 \alpha_{2} b \boldsymbol{q}^{\prime \prime}+\alpha_{1} \alpha_{2} \boldsymbol{q}^{\prime} / 4+ \\
& +3 \alpha_{1}^{2} \boldsymbol{q}^{\prime \prime} / 4+3 \alpha_{1} b \boldsymbol{q}^{\prime \prime \prime}+\boldsymbol{q}^{\mathrm{iv}} b^{2} .
\end{aligned}\right.
$$

These equations can be rewritten as a linear combination of a set of functions $\beta_{i}$ as follows

$$
\begin{aligned}
\dddot{\boldsymbol{q}}= & \boldsymbol{q}^{\prime} \beta_{1} / 2+(3 / 2) \boldsymbol{q}^{\prime \prime} \beta_{2}+\boldsymbol{q}^{\prime \prime \prime} \beta_{3}, \\
\boldsymbol{q}^{(4)}= & (1 / 2) \boldsymbol{q}^{\prime} \beta_{4}+2 \mathbf{q}^{\prime \prime} \beta_{5}+(1 / 4) \boldsymbol{q}^{\prime} \beta_{6}+ \\
& +(3 / 4) \boldsymbol{q}^{\prime \prime} \beta_{7}+3 \mathbf{q}^{\prime \prime \prime} \beta_{8}+\boldsymbol{q}^{\text {iv }} \beta_{9},
\end{aligned}
$$

where we define

$$
\begin{aligned}
& \beta_{1} \triangleq \alpha_{2} \sqrt{b}, \beta_{2} \triangleq \alpha_{1} \sqrt{b}, \beta_{3} \triangleq b \sqrt{b}, \beta_{4} \triangleq \alpha_{3} b, \beta_{5} \triangleq \alpha_{2} b, \\
& \beta_{6} \triangleq \alpha_{1} \alpha_{2}, \beta_{7} \triangleq \alpha_{1}^{2}, \beta_{8} \triangleq \alpha_{1} b, \beta_{9} \triangleq b^{2}, \beta_{10} \triangleq \sqrt{b} .
\end{aligned}
$$

With a procedure similar to the one proposed in this work, it is possible to write the time-optimal rest-to-rest problem with bounds up to the snap

$$
\begin{aligned}
& \min _{b(\cdot), \beta_{i}(\cdot), \alpha_{i}(\cdot)} \int_{0}^{1} \frac{1}{\sqrt{b}} \mathrm{~d} s \\
& \text { subject to } \\
& b(0)=0 \text { and } b(1)=0 \\
& \alpha_{1}(0)=0 \text { and } \alpha_{1}(1)=0 \\
& \alpha_{2}(0) \sqrt{b(0)}=0 \text { and } \alpha_{2}(1) \sqrt{b(1)}=0 \\
& \alpha_{1}=b^{\prime}, \alpha_{2}=b^{\prime \prime}, \alpha_{3}=b^{\prime \prime \prime} \\
& \beta_{1}=\alpha_{2} \beta_{10}, \beta_{2}=\alpha_{1} \beta_{10}, \beta_{3}=b \beta_{10}, \beta_{4}=\alpha_{3} b \\
& \beta_{5}=\alpha_{2} b, \beta_{6}=\alpha_{1} \alpha_{2}, \beta_{8}=\alpha_{1} b \\
& \beta_{7}=\alpha_{1}^{2}, \beta_{9}=b^{2}, \beta_{10}^{2}=b \\
& 0<b \leq \min _{i}\left(\overline{\dot{q}}_{i}\right)^{2} /\left(q_{i}^{\prime}\right)^{2} \\
& \ddot{\boldsymbol{q}} \leq \boldsymbol{q}^{\prime \prime} b+\boldsymbol{q}^{\prime} \alpha_{1} / 2 \leq \ddot{\boldsymbol{q}} \\
& \dddot{\ddot{q}} \leq \boldsymbol{q}^{\prime} \beta_{1}+(3 / 2) \boldsymbol{q}^{\prime \prime} \beta_{2}+\boldsymbol{q}^{\prime \prime \prime} \beta_{3}, \leq \overline{\ddot{q}} \\
& \underline{\boldsymbol{q}}^{(4)} \leq \boldsymbol{q}^{\prime} \beta_{4} / 2+2 \mathbf{q}^{\prime \prime} \beta_{5}+\boldsymbol{q}^{\prime} \beta_{6} / 4+ \\
& \quad+(3 / 4) \boldsymbol{q}^{\prime \prime} \beta_{7}+3 \mathbf{q}^{\prime \prime \prime} \beta_{8}+\boldsymbol{q}^{\text {iv }} \beta_{9} \leq \overline{\boldsymbol{q}}^{(4)} \\
& \text { for } s \in[0,1] .
\end{aligned}
$$

The constraints can be divided in differential constraints, constraints that are linear in the set of variables $b, \alpha_{i}, \beta_{i}$ and, again, a set of bilinear and quadratic constraints.

\section{REFERENCES}

[1] C. Laschi, B. Mazzolai, and M. Cianchetti. Soft robotics: Technologies and systems pushing the boundaries of robot abilities. Science Robotics, $1(1), 2016$.
[2] C. Della Santina, C. Piazza, G. M. Gasparri, M. Bonilla, M. G. Catalano, G. Grioli, M. Garabini, and A. Bicchi. The quest for natural machine motion: An open platform to fast-prototyping articulated soft robots. IEEE Robotics and Automation Mag., 24(1):48-56, 2017.

[3] M. Hutter et al. Anymal - a highly mobile and dynamic quadrupedal robot. In Proc. IEEE/RSJ Int. Conf. on Intelligent Robots and Systems, pages $38-44,2016$.

[4] N. Tsagarakis et al. Walk-man: A high-performance humanoid platform for realistic environments. J. of Field Robotics, 34(7):1225-1259, 2017.

[5] D. J. Braun, F. Petit, F. Huber, S. Haddadin, P. van der Smagt, A. AlbuSchäffer, and S. Vijayakumar. Robots driven by compliant actuators: Optimal control under actuation constraints. IEEE Trans. on Robotics, 29(5):1085-1101, 2013.

[6] A. Zhakatayev, M. Rubagotti, and H. Atakan Varol. Time-optimal control of variable-stiffness-actuated systems. IEEE/ASME Trans. on Mechatronics, 22(3):1247-1258, 2017.

[7] C. Ji, M. Kong, and R. Li. Time-energy optimal trajectory planning for variable stiffness actuated robot. IEEE Access, 7:14366-14377, 2019.

[8] T. Marcucci, M. Garabini, G. M. Gasparri, A. Artoni, M. Gabiccini, and A. Bicchi. Parametric trajectory libraries for online motion planning with application to soft robots. In 18th Int. Symp. on Robotics Research, 2017.

[9] G. M. Gasparri, S. Manara, D. Caporale, G. Averta, M. Bonilla, H. Marino, M. Catalano, G. Grioli, M. Bianchi, A. Bicchi, and M. Garabini. Efficient walking gait generation via principal component representation of optimal trajectories: Application to a planar biped robot with elastic joints. IEEE Robotics and Automation Lett., 3(3):22992306, 2018.

[10] K. G. Shin and N. D. McKay. Minimum-time control of robotic manipulators with geometric path constraints. IEEE Trans. on Automatic Control, 30(6):531-541, 1985.

[11] D. Verscheure, B. Demeulenaere, J. Swevers, J. De Schutter, and M. Diehl. Time-optimal path tracking for robots: A convex optimization approach. IEEE Trans. on Automatic Control, 54(10):2318-2327, 2009.

[12] T. Lipp and S. P. Boyd. Minimum-time speed optimisation over a fixed path. Int. J. Control, 87(6):1297-1311, 2014.

[13] H. Pham and Q.-C. Pham. A new approach to time-optimal path parameterization based on reachability analysis. IEEE Trans. on Robotics, 34(3):645-659, 2018.

[14] A. Palleschi, M. Garabini, D. Caporale, and L. Pallottino. Time-optimal path tracking for jerk controlled robots. IEEE Robotics and Automation Lett., 4(4):3932-3939, 2019.

[15] M. Ruf. Near time-optimal flexible-joint trajectory planning algorithm for robotic manipulators. In Proc. IEEE Conf. on Control Technology and Applications, pages 265-272, 2018.

[16] G. P. McCormick. Computability of global solutions to factorable nonconvex programs: Part I-Convex underestimating problems. Mathematical Programming, 10(1):147-175, 1976.

[17] M. W. Spong. Modeling and control of elastic joint robots. ASME J. of Dynamic Systems, Measurement, and Control, 109(4):310-318, 1987.

[18] P. Belotti, J. Lee, L. Liberti, F. Margot, and A. Wächter. Branching and bounds tightening techniques for non-convex MINLP. Optimization Methods and Software, 24(4-5):597-634, 2009.

[19] L. Liberti and C. C. Pantelides. Convex envelopes of monomials of odd degree. J. of Global Optimization, 25(2):157-168, 2003.

[20] R. Misener and C. A. Floudas. ANTIGONE: Algorithms for continuous I integer global optimization of nonlinear equations. J. of Global Optimization, 59(2):503-526, 2014.

[21] J. Lofberg. YALMIP: A toolbox for modeling and optimization in MATLAB. In Proc. IEEE Int. Conf. on Robotics and Automation, pages 284-289, 2004.

[22] K. Springer, H. Gattringer, and P. Staufer. On time-optimal trajectory planning for a flexible link robot. Proc. of the Institution of Mechanical Engineers, Part I: J. of Systems and Control Engineering, 227(10):752763, 2013.

[23] A. De Luca and P. Lucibello. A general algorithm for dynamic feedback linearization of robots with elastic joints. In Proc. IEEE Int. Conf. on Robotics and Automation, pages 504-510, 1998. 